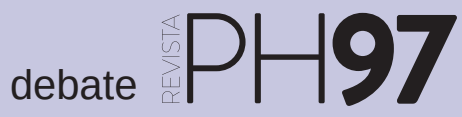

a debate El papel del patrimonio en las nuevas políticas para la cultura, la ciudad y el territorio:

las agendas urbanas como marco de oportunidad

| coordinan Blanca del Espino Hidalgo, Rafael Merinero Rodríguez

\title{
La cultura y el patrimonio como factores de desarrollo sostenible
}

\author{
Felisa Becerra Benítez | Analistas Económicos de Andalucía \\ URL de la contribución <http://www.iaph.es/revistaph/index.php/revistaph/article/view/4380>
}

Efectivamente, las distintas estrategias de desarroIlo puestas en marcha en los últimos años (Estrategia 2020 Europa, Objetivos de Desarrollo Sostenible) parecen no abordar de forma expresa el papel de la cultura y el patrimonio como elementos esenciales para un desarrollo equilibrado y sostenible, sino que estos elementos tienen quizás un carácter transversal a los distintos objetivos que se persiguen con estas estrategias.

Sin embargo, no cabe duda de que implícitamente se reconoce el potencial de la cultura en la construcción de sociedades más inclusivas, justas y solidarias, y su aportación a la innovación, la creatividad, el empleo y el crecimiento sostenible, pilares centrales de la Estrategia Europa 2020. La cultura y el patrimonio resultan esenciales para el desarrollo urbano sostenible, ayudando a la revitalización de áreas degradadas y el fortalecimiento de la participación social, algo que estaría implícito en el objetivo 11 de la Agenda 2030 para el Desarrollo Sostenible ("Conseguir que las ciudades y los asentamientos humanos sean inclusivos, seguros, resilientes y sostenibles").

Por su parte, en el 60 aniversario de los Tratados de Roma en 2017, los Estados miembros e instituciones de la Unión Europea señalaron su apuesta por una Unión "en la que los ciudadanos tengan nuevas oportunidades de desarrollo cultural y social y de crecimiento económico [...] una Unión que conserve nuestro patrimonio cultural y promueva la diversidad cultural". En opinión de la Comisión Europea es importante tomar conciencia de la importancia social y económica de la cultura y el patrimonio, dado que la cultura fomenta la ciudadanía activa, la inclusión, los valores comunes o el diálogo intercultural, al tiempo que las industrias culturales tienen capacidad para generar riqueza y empleo y mejorar la calidad

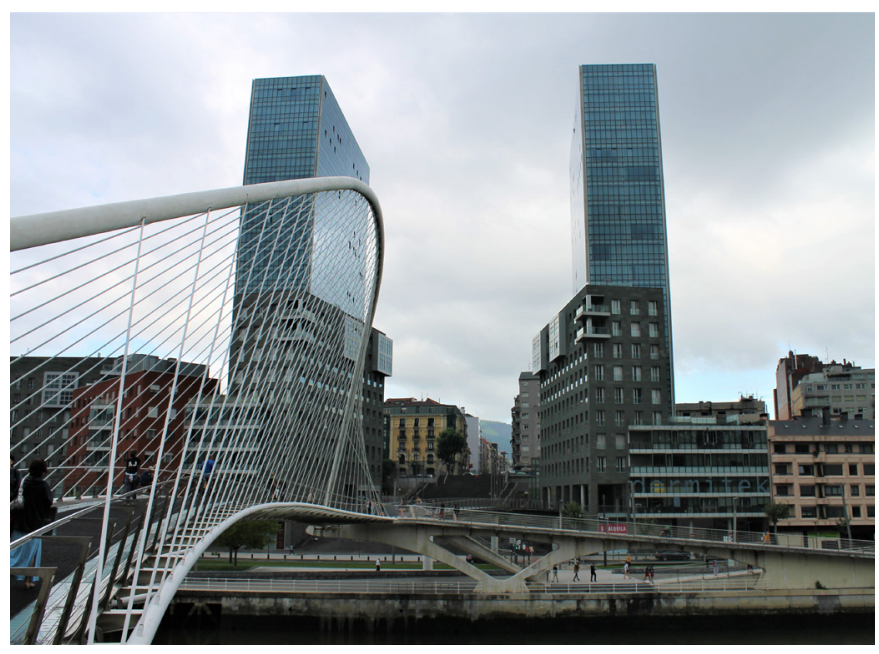

Ría Nervion, , Bilbao (agosto, 2016) | foto Blanca del Espino Hidalgo

de vida de los ciudadanos. Todo esto se plasma en la Nueva Agenda Europea para la cultura (presentada en mayo del pasado año).

Por tanto, la relevancia y el papel de la cultura y el patrimonio como impulsores del desarrollo sostenible parece clara para las administraciones públicas y organismos internacionales. Al mismo tiempo, los ciudadanos de la UE creen que la cultura es el factor más importante en la creación de un sentido de comunidad, por lo que no cabe duda de que las agendas urbanas están llamadas a convertirse en un instrumento primordial para la puesta en valor de estos activos.

Si bien es cierto que estas agendas no tienen carácter normativo, hay experiencias que evidencian el éxito de contar con planes estratégicos a largo plazo, que permitan definir el modelo de ciudad que se quiere y diseñar el futuro de la misma (casos de Bilbao, Málaga o Zaragoza). 
a debate El papel del patrimonio en las nuevas políticas para la cultura, la ciudad y el territorio: las agendas urbanas como marco de oportunidad

| coordinan Blanca del Espino Hidalgo, Rafael Merinero Rodríguez

Obviamente el hecho de ser instrumentos de adhesión voluntaria supone un reto a la hora de intentar poner en marcha estas iniciativas, para lo que sin duda la participación de los distintos agentes económicos y sociales resulta crucial. Evidentemente en aquellos casos en los que se ha realizado un verdadero esfuerzo porque todos los agentes estuviesen implicados en los procesos de diagnóstico y planificación de líneas de actuación los resultados son y serán más satisfactorios.

En mi opinión, el caso de la ciudad de Málaga puede ser un ejemplo de participación y éxito de la planificación estratégica, registrando la ciudad una profunda transformación en las últimas décadas. Ya el primer plan estratégico de la ciudad de 1992-1996 incluía cuatro líneas estratégicas, ampliadas posteriormente a cinco, a una de las cuales le concernían los temas culturales, turísticos y comerciales.

Posteriormente, en 2002 se decidió poner en marcha el segundo plan estratégico, estructurado en este caso en torno a cuatro ejes de actuación y diez grandes proyectos estrella, entre ellos “Ágora Mediterráneo: La ciudad como espacio de cultura". Más recientemente, la Estrategia Europea Horizonte 2020 se ha convertido en una referencia para la estrategia territorial, al igual que los objetivos de desarrollo sostenible, con una de las líneas centradas en la "Málaga de la cultura".

Precisamente, en la Estrategia Málaga 2020 se considera que la cultura debe seguir siendo un elemento dinamizador de la economía, la sociedad y el desarrollo urbano, y se señala como prioridad extender la ciudad como espacio de cultura hacia otros enclaves al margen del centro histórico. Sin duda, esta puede ser una de las principales críticas en cuanto al desarrollo de la ciudad, común a otros territorios, como es el hecho de que el proceso de regeneración urbana y el impulso de la actividad, en este caso ligado a la apuesta por la cultura, se aprecia en mayor medida en el centro histórico, por lo que su extensión a otros enclaves resulta primordial para el equilibrio territorial y el desarrollo inclusivo y sostenible de la ciudad.
Además, esta extensión tendría también ventajas para el modelo de planificación turística. El turismo de masas como tal estaría reñido con el modelo de desarrollo sostenible que proponen las agendas urbanas, de ahí que la mayor parte de las administraciones locales se esfuercen en impulsar modelos de desarrollo turístico sostenibles. En este sentido, la cultura y el patrimonio suponen un referente y una apuesta de las ciudades, ya que su puesta en valor constituye un gran atractivo para las mismas, aunque no hay que olvidar la necesidad de conciliar la atracción turística con el disfrute de los ciudadanos que habitan las ciudades. Además, hay que tener en cuenta la importancia de proteger y conservar el patrimonio cultural, y si bien es cierto que en los últimos años se están produciendo avances en este sentido, también lo es la necesidad de dedicar más esfuerzos e inversión a cuidar y conservar este patrimonio.

En definitiva, y aunque la cultura y el patrimonio no aparezcan como líneas u objetivos de las actuales estrategias de desarrollo, su carácter transversal y su potencial para impulsar sociedades más inclusivas, creativas e innovadoras no dejan lugar a dudas sobre la necesidad de incluirlas en la planificación estratégica y las agendas urbanas. Si bien es cierto que estas agendas tuvieron quizá en principio una dimensión más económica, con objetivos más vinculados a lograr un crecimiento económico sostenible, ahora cobra también mucha importancia la dimensión social (personas, alianzas...). Este foco también en las personas o las alianzas es el que invita a pensar que las agendas urbanas, centradas en los territorios que acogen a una mayor parte de la población, pueden ser compatibles con el impulso de los territorios rurales, no solo porque a veces el medio rural o municipios de pequeño tamaño graviten en torno a ciudades de tamaño medio (como ocurre en Andalucía) sino también porque el desarrollo sostenible no se entendería sin el equilibrio y la cohesión territorial. 
_a debate El papel del patrimonio en las nuevas políticas para la cultura, la ciudad y el territorio: las agendas urbanas como marco de oportunidad

| coordinan Blanca del Espino Hidalgo, Rafael Merinero Rodríguez

\section{BIBLIOGRAFÍA}

- AGENDA Urbana de Andalucía 2030 (2018) Consejería de Medio Ambiente y Ordenación del Territorio, 2018

- CULTURA urbana y patrimonio (2015). Naciones Unidas, 31 de mayo de 2015. Documento inédito para la Conferencia de las Naciones Unidas sobre la Vivienda y el Desarrollo Urbano Sostenible (Hábitat III, Quito, Octubre 2016)

- ESTRATEGIA Málaga 2020. Prioridades para un trabajo conjunto (2017) Fundación CIEDES, 2017

- EUROPA 2020. Una estrategia para un crecimiento inteligente, sostenible integrador (2010) Comisión Europea, 2010, 2020 final

- Jornada de debate y presentación de la Agenda Urbana española. Marco estratégico para orientar las políticas urbanas (2018) Ministerio de Fomento, abril 2018

- MIRANDO hacia el futuro: Ciudades Sostenibles. Los Objetivos de Desarrollo Sostenible en 100 ciudades españolas (2018) Informe urbano. Red Española para el Desarrollo Sostenible, 2018

- Una NUEVA Agenda europea para la cultura (2018) Comisión Europea, 2018, 267 final 